\title{
riccafd
}

Revista Iberoamericana de Ciencias de la Actividad Física y el Deporte

\section{DISFRUTE A TRAVÉS DE UNA PROPUESTA DE DESCANSOS ACTIVOS EN EDUCACIÓN INFANTIL}

\section{ENJOYMENT THROUGH A PROPOSAL OF ACTIVE BREAKS IN EARLY CHILDHOOD EDUCATION}

Montoya-Fernández, C1ABCD; Zamorano-García, D ${ }^{2 A C D F}$; Gil-Madrona, P ${ }^{3 A C D}$; Infantes-Paniagua, $A^{4 A C D}$

${ }^{1}$ Estudiante del Máster Universitario en Educación Física en Educación Primaria y Deporte en Edad Escolar, Universidad de Castilla - La Mancha, España, Carlos.Montoya@alu.uclm.es

${ }^{2}$ Profesor Asociado, Universidad de Castilla - La Mancha, España, David.Zamorano@uclm.es

${ }_{3}$ Profesor Titular, Universidad de Castilla - La Mancha, España, Pedro.Gil@uclm.es

${ }^{4}$ Estudiante de Doctorado, Universidad de Castilla - La Mancha, España, Alvaro.Infantes@uclm.es

\section{Responsabilidades}

A Diseño de la investigación

B Recolector de datos

C Redactor del trabajo

D Tratamiento estadístico

E Apoyo económico

$\mathrm{F}$ Idea original y coordinador de toda la investigación

Recibido el 5 de enero de 2020

Aceptado el 26 de febrero de 2020

Correspondencia: Zamorano, D David.Zamorano@uclm.es

DOI: http://dx.doi.org/10.24310/riccafd.2020.v9i1.8305

\section{RESUMEN}

La siguiente propuesta muestra un estudio que pretendió comprobar cuál es la influencia que tienen los descansos activos en el disfrute del alumnado de Educación Infantil, siendo este un elemento de la motivación. Además, se evaluó la existencia de diferencias en el disfrute en función del sexo del alumnado. Para ello, se llevó a cabo una metodología basada en los descansos activos durante diez días, en los cuales se recogieron las impresiones del alumnado a través de un test que se pasaba tras la actividad sedentaria, y, de igual forma, tras los descansos activos. Igualmente, se recogieron las impresiones por parte del profesorado a través de una entrevista realizada el último día del estudio. La muestra tomada para este trabajo constaba de 22 sujetos de una clase del primer curso del segundo ciclo de Educación Infantil (3 años), siendo estos 12 niñas y 10 niños. Finalmente, los resultados permitieron concluir que los descansos activos aumentan el disfrute del alumnado, sin que se den diferencias de acuerdo con la variable sexo. 
Palabras clave: descansos activos, disfrute, motivación, Educación Infantil.

\begin{abstract}
The following proposal shows a study that attempted to verify the influence that active rests have on the enjoyment of Infant Education students, this being an element of motivation. In addition, the existence of differences in enjoyment according to the sex of the student body was evaluated. To this end, a methodology was carried out based on active breaks during ten days, in which the student's impressions were collected through a test that was passed after sedentary activity and, in the same way, after active breaks. Likewise, the impressions were collected from the teachers through an interview carried out on the last day of the study. The sample taken for this work consisted of 22 subjects from a class in the first year of the second cycle of Infant Education ( 3 years), these being 12 girls and 10 boys. Finally, the results allowed us to conclude that active breaks increase the enjoyment of the students, without differences according to the sex variable.
\end{abstract}

Key words: active breaks, enjoyment, motivation, Early Childhood Education.

\title{
INTRODUCCIÓN
}

El disfrute es un concepto complejo y variado cuya definición abarca enormes campos asociados a multitud de disciplinas y ámbitos, como el afectivo, conductual, psicológico, deportivo, etc. Igualmente, es un elemento asociado a la motivación, y más concretamente, a la motivación intrínseca, como proceso regulador de la misma (1). Puede definirse como un concepto que no es aislado e independiente, sino asociado a diversos ámbitos, a ideas, la autoestima o, por supuesto, a la motivación; e incluso a experiencias, como la deportiva. Todo ello da una idea sobre el enorme y variado campo que abarca el disfrute, y la manera en que un individuo puede llegar a alcanzar ese estado $(2,3,4,5,6)$. Por ello, para poder hablar del disfrute, primero se debe de hacer un recorrido a través del significado de la palabra motivación. Atendiendo a los autores citados con anterioridad, se podría resumir que la motivación es la fuerza que mueve la conducta humana para realizar una acción. Asimismo, la mayoría distinguen que la motivación se divide en dos tipos, intrínseca y extrínseca $(7,8,9)$.

Por otro lado, el elemento en torno al cual se desarrollará este trabajo serán los descansos activos, los cuales son una metodología basada en cortos periodos de actividad física media-vigorosa implementados en distintos momentos de la jornada escolar, con el objetivo de mejorar distintas facetas del alumnado, como disminuir el tiempo de sedentarismo, reducir las conductas disruptivas, aumentar la diversión, la función cognitiva, el rendimiento escolar, etc. (10). Una herramienta para llevarlos a cabo de manera eficaz es GoNoodle, una web que provee de recursos útiles, sencillos y divertidos para la puesta en marcha de esta propuesta metodológica. Todo ello a través de cientos de vídeos a 
disposición del profesorado para dirigir la energía del alu,nado, a la vez que innovan y se distancian de metodologías tradicionales de enseñanza en busca de una propuesta divertida y entretenida (11). Ésta se centra en un proceso de investigación y aplicación, fundamentada en estudios relacionados que se han encargado de evaluar los cambios fisiológicos, conductuales, cognitivos, académicos, etc., que los descansos activos producen. Sin embargo, pocos se centran en atender a los cambios producidos en cuanto al disfrute del alumnado, siendo este elemento muy relevante para aumentar la motivación en el aula.

Ante el panorama actual, este trabajo pretende dar una primera respuesta a la forma en la que los descansos activos influyen en el disfrute del alumnado de Educación Infantil. Además, se pretende también verificar si existen diferencias en cuanto al disfrute del alumnado en función de la variable sexo.

Para poder llevarlo a cabo, se utilizó un instrumento de evaluación de elaboración propia que parte de la propuesta de la Escala de Disfrute con la Actividad Física (PACES) (12) así como de la propuesta metodológica de la Escala de la Motivación hacia el Aprendizaje Infantil (EMAPI) (13). Además, para dotar a la evaluación de una mayor riqueza y perspectiva, se pasó una encuesta a la tutora del curso, a la maestra de Inglés, y al maestro de Educación Física, los cuales aportaron su visión acerca de cómo habían influido los descansos activos al disfrute del alumnado.

El estudio duró diez días, en los que, inicialmente, se pasaba un primer test al alumnado tras la actividad sedentaria, seguidamente se realizaban los descansos activos, y por último se pasaba nuevamente el test.

Los resultados obtenidos tras los días de estudio permiten afirmar que, efectivamente, el alumnado mostraba un aumento del disfrute tras la realización de los descansos activos, sin apreciar diferencias con respecto al sexo del alumnado.

\section{MATERIAL Y METODOS}

\section{Participantes}

La muestra disponible para este estudio es de 22 sujetos; 12 niñas y 10 niños de un centro educativo de Educación Infantil y Primaria, concretamente del primer curso del Segundo Ciclo de Educación Infantil (3 años). El alumnado participa en clases de Educación Física durante 45 minutos una vez por semana, sin apenas práctica de actividad físico-deportiva estructurada a nivel extracurricular.

\section{Instrumentos de recogida de datos}

Se ha hecho uso de un instrumento de elaboración propia que partiendo del contenido de la Escala de Disfrute con la Actividad Física (PACES) (12), 
y adaptándolo a las características propias del momento de desarrollo del alumnado en cuestión. De igual forma, la manera de presentar el instrumento, de proceder y de evaluar será conforme a la escala EMAPI (13). Además, se acompañará a cada ítem de una explicación y una ejemplificación oral para mejorar la comprensión de estos por parte de los niños y niñas, y ayudar así a que las respuestas sean lo más coherentes posible.

Para su implementación, se ha acompañado a los ítems de evaluación de una imagen representativa de cada uno. Con cada ítem, se presentarán dos opciones de posibles respuestas al alumnado, el cual deberá de seleccionar una de ellas para contestar a las afirmaciones. Esto permitirá a los niños y niñas identificar mejor su respuesta, y tener los conceptos que se desean evaluar más claros, facilitando la comprensión y la calidad de la respuesta. Asimismo, se utilizará una encuesta que permita que el profesorado que está con el alumnado pueda evaluar de igual manera el disfrute de estos, y obtener un registro con otro tipo de perspectiva que pueda dotar a la evaluación de una mayor riqueza.

\section{Procedimiento}

Procedimientos generales previos a la investigación

Para poder realizar este trabajo, el primer paso supuso solicitar permiso al equipo directivo del centro, Consejo Escolar, y tutora de la clase, maestra de Inglés y maestro de Educación Física. Todos dieron su consentimiento para el desarrollo de la propuesta. El estudio se realizó durante dos semanas. El momento idóneo fue entre tareas que requerían una actitud sedentaria, y estuvo marcado por el desarrollo de cada jornada.

Procedimiento de administración de test y entrevista

Se llamó individualmente a cada alumno para que realizase el cuestionario tras un periodo de actividad sedentaria. Al finalizar, se procedía a la práctica del descanso activo. Posterior a ello, se volvía a pasar la prueba. Todo este proceso, duraba alrededor de 30 minutos. El último día se entregó la entrevista a la tutora, a la maestra de Inglés y al maestro de Educación Física, los cuales dispusieron del tiempo que estimaron oportuno para contestar.

Procedimiento de desarrollo de los descansos activos

Los descansos activos se realizaron, generalmente, entre la segunda y la tercera sesión de clase. Tras pasar el primer test a cada individuo, se les incentivaba a abandonar lo que estuvieran haciendo para reunirse en el sitio donde se realizaba la asamblea diariamente. Una vez allí, y consiguiendo captar la atención del alumnado, se desarrollaba el descanso activo, con una duración aproximada de entre 5 y 10 minutos. 


\section{RESULTADOS}

En la siguiente tabla, se muestran los resultados obtenidos tras el primer test (tras la actividad sedentaria) y el segundo test (tras descansos activos). Se aprecian, por un lado, las respuestas positivas y negativas expresadas por cada sujeto de la muestra, tanto tras la actividad sedentaria como tras los descansos activos; y por el otro, se puede ver el total de respuestas positivas y negativas del conjunto del alumnado, para cada uno de los test, incluyendo el porcentaje que supone respecto al total de respuestas emitidas.

Tabla 1: Resultados del primer test y el segundo test.

\begin{tabular}{|c|c|c|c|c|}
\hline \multirow[b]{2}{*}{ Alumnado } & \multicolumn{2}{|c|}{ Primer test } & \multicolumn{2}{|c|}{ Segundo test } \\
\hline & $\begin{array}{c}\text { Respuestas } \\
\text { positivas }\end{array}$ & $\begin{array}{c}\text { Respuestas } \\
\text { negativas }\end{array}$ & $\begin{array}{l}\text { Respuestas } \\
\text { positivas }\end{array}$ & $\begin{array}{c}\text { Respuestas } \\
\text { negativas }\end{array}$ \\
\hline Alumna 1 & 38 & 2 & 40 & 0 \\
\hline Alumno 2 & 40 & 0 & 40 & 0 \\
\hline Alumno 3 & 36 & 4 & 40 & 0 \\
\hline Alumna 4 & 39 & 1 & 40 & 0 \\
\hline Alumno 5 & 29 & 11 & 40 & 0 \\
\hline Alumna 6 & 40 & 0 & 40 & 0 \\
\hline Alumno 7 & 31 & 5 & 36 & 0 \\
\hline Alumno 8 & 40 & 0 & 40 & 0 \\
\hline Alumna 9 & 40 & 0 & 40 & 0 \\
\hline Alumno 10 & 40 & 0 & 39 & 1 \\
\hline Alumna 11 & 36 & 0 & 36 & 0 \\
\hline Alumna 12 & 26 & 14 & 40 & 0 \\
\hline Alumno 13 & 34 & 6 & 40 & 0 \\
\hline Alumna 14 & 33 & 3 & 36 & 0 \\
\hline Alumna 15 & 29 & 3 & 31 & 1 \\
\hline Alumno 16 & 39 & 1 & 40 & 0 \\
\hline Alumno 17 & 19 & 21 & 40 & 0 \\
\hline Alumna 18 & 36 & 4 & 40 & 0 \\
\hline Alumno 19 & 36 & 0 & 36 & 0 \\
\hline Alumna 20 & 28 & 0 & 28 & 0 \\
\hline Alumna 21 & 24 & 8 & 32 & 0 \\
\hline Alumna 22 & 20 & 16 & 36 & 0 \\
\hline Media aritmética & 33,32 & 4,5 & 37,73 & 0,09 \\
\hline Total & 733 (88,1\%) & 99 (11,9\%) & $830(99,76 \%)$ & $2(0,24 \%)$ \\
\hline
\end{tabular}


De igual forma, a través del siguiente gráfico se puede apreciar una comparativa de los datos expuestos en la tabla anterior, donde se comparan de forma clara las respuestas positivas obtenidas en el primer test con las obtenidas en el segundo test.

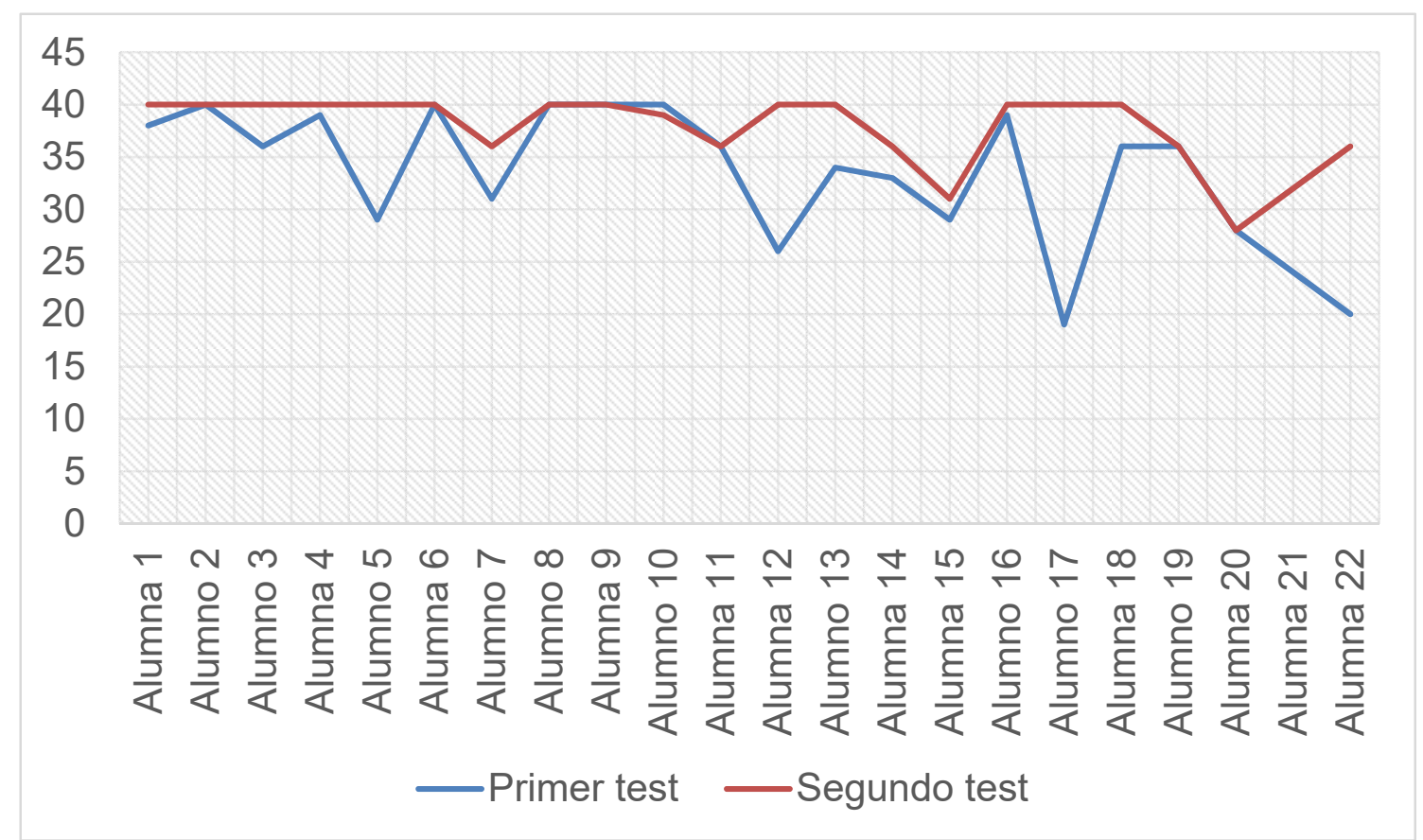

Figura 1. Comparativa de respuestas positivas entre el primer test y el segundo test.

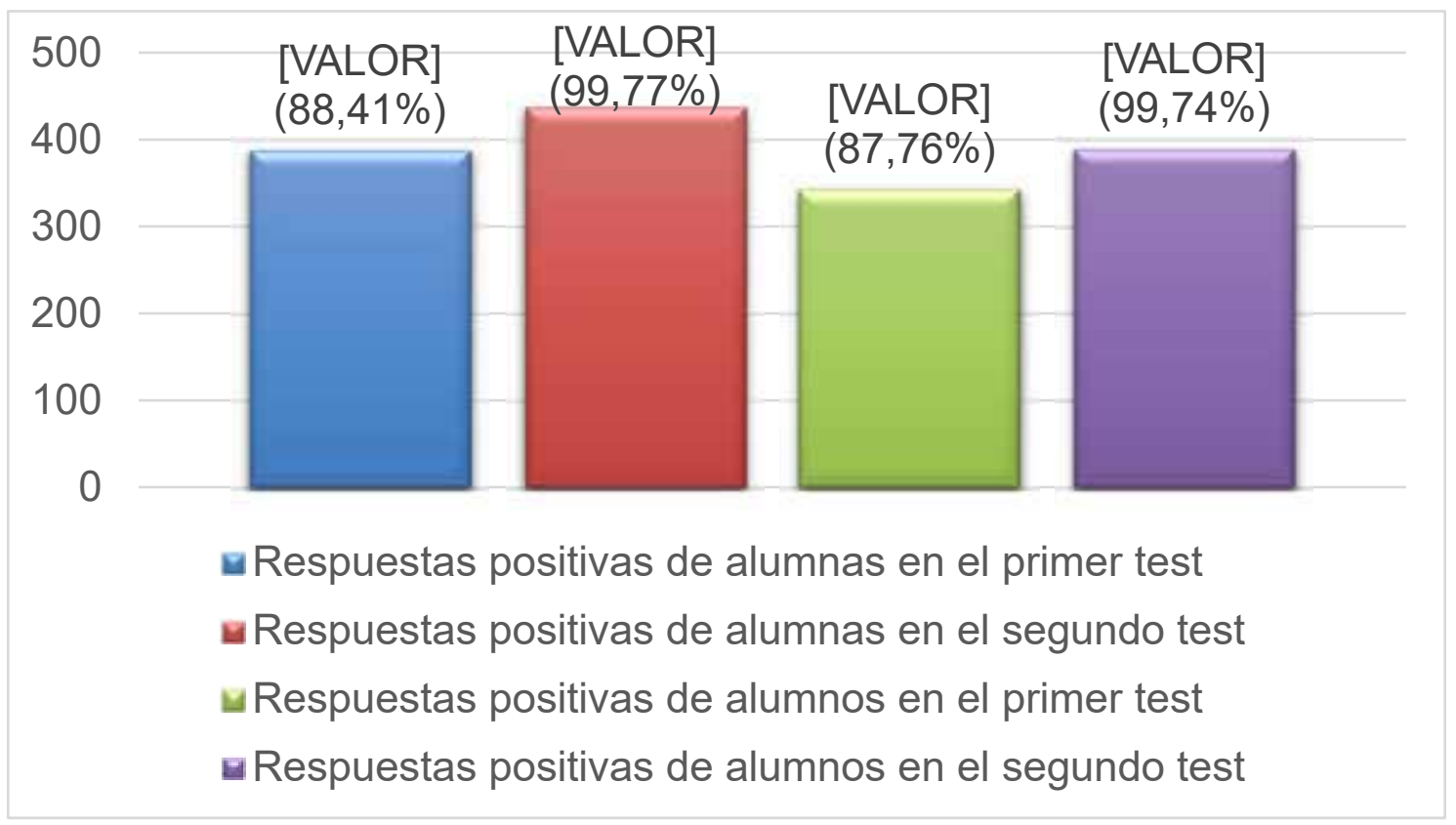

Fig. 2. Comparativa de respuestas positivas entre alumnos y alumnas.

De acuerdo con el objetivo general que habla de evaluar el efecto de los descansos activos en el disfrute del alumnado de Educación Infantil, se puede ob- 
servar un aumento de respuestas positivas en la mayoría del alumnado. De esta forma, se confirma la hipótesis que se refiere a que la práctica de actividad física a través de descansos activos produce mejoras en el disfrute del alumnado.

A continuación, pueden apreciarse los resultados obtenidos en cuanto a respuestas positivas considerando la variable sexo.

En relación con el objetivo referido a valorar la existencia de diferencias en el disfrute en función de la variable sexo, por lo general, se han obtenido resultados similares en cuanto a niños y niñas, siendo el número de respuestas positivas escasamente mayor en las niñas. Por un lado, el porcentaje de respuestas positivas de estas en el primer test es del 88'41\%, mientras que el de los niños es de $87^{\prime} 76 \%$. Sin embargo, en el segundo test, los resultados arrojan un 99'77\% de respuestas positivas en las niñas, por el 99'74\% de los niños. Por todo ello, se puede apreciar que la mejora es algo superior en los alumnos, siendo esta de un $11^{\prime} 98 \%$, mientras que en las alumnas es de un 11 '36\%. Estos resultados ayudan a confirmar la hipótesis referida a la no existencia de diferencias en función del sexo.

Igualmente, puede decirse que tanto niños como niñas han obtenido, de igual manera, una mejora en su disfrute a través de los descansos activos, siendo las diferencias prácticamente inapreciables.

Tabla 2: Entrevista a los docentes.

$\begin{array}{lll}\text { Preguntas } & \text { Tutora } & \begin{array}{c}\text { Maestro de } \\ \text { Educación Física }\end{array} \\ \text { Maestra de Inglés }\end{array}$

1. ¿Consideras que el alumnado disfruta más que antes de realizar el proyecto de descansos activos? ¿Por qué?

Sí

Sí

Sí

2. ¿Crees que el alumnado encuentra agradables los descansos activos? ¿Por qué? alumnado?

4. ¿Observas que los descansos activos producen sentimientos positivos en el alumnado? ¿Cómo lo manifiestan?

5. ¿Piensas que el alumnado encuentra motivante y excitante la realización de los descansos activos?

6. ¿Observas que el alumnado se siente más motivado tras la realización de los descansos activos?

7. ¿Hay diferencias en cuanto al disfrute entre niños y niñas a través de los descansos activos?
Sí

No

$\begin{array}{lll}\text { Sí } & \text { Sí } & \text { Sí } \\ \text { No } & \text { No } & \text { No } \\ \text { Sí } & \text { Sí } & \text { Sí }\end{array}$

Sí

Sí

Sí

Sí

Sí

Sí

No

No

No 
Los docentes coinciden en señalar que el alumnado disfruta más tras realizar los descansos activos, e incluso que es un momento esperado con alegría por la clase. Creen además que los descansos activos resultan agradables al alumnado, y que en ningún caso les produce frustración, sino todo lo contrario. Una de las maestras llegó a destacar que estos han influido positivamente en las interacciones que realiza el alumnado entre sí, mejorando las relaciones sociales en el grupo. Hay consenso al afirmar que este método produce sentimientos positivos en el alumnado, manifestándose alegres y sin percibir cansancio en ellos por la práctica de esta actividad. Observan una motivación extra en el alumnado, sirviéndoles para recuperar fuerzas y llevándolos a encarar los trabajos y actividades posteriores de manera positiva, resultándoles excitante la práctica de los descansos activos. De igual forma, esa motivación que manifiestan a la hora de realizar los descansos activos se prolonga a lo largo de la jornada. Por último, los docentes no encuentran diferencias en cuanto al disfrute entre niños y niñas.

\section{DISCUSIÓN}

El efecto beneficioso de la práctica de actividad física durante los descansos activos puede deberse a diferentes cambios fisiológicos, físicos e incluso ambientales/sociales que tienen lugar durante y tras la intervención (14). La actividad física media-vigorosa incrementa la práctica de actividad física, capacidad que influye directamente en la función cerebral (15). De igual forma, la práctica de actividad física aumenta el factor neurotrófico derivado del cerebro, que regula la supervivencia celular, la diferenciación y la plasticidad del cerebro (16), lo que supone una mejora en la función cognitiva (17). El disfrute está relacionado con numerosos factores, entre ellos la función cognitiva, por lo que se podría considerar que ese aumento de la función cognitiva, junto con otras variables, podría desencadenar en un aumento de la capacidad de disfrute.

Hay autores que mencionan mejoras importantes en variables cognitivas tras la realización del programa, así como mejoras en el comportamiento, algo que se puede relacionar con el disfrute, ampliamente vinculado a factores conductuales (18). Así, una mejora en la conducta a través de los descansos activos podría conllevar una mejora en el disfrute.

\section{LIMITACIONES Y CAMINOS FUTUROS}

Por supuesto, hemos de considerar las limitaciones que se han encontrado y, entre las que destacan la escasez de estudios que relacionen de forma directa cómo afectan los descansos activos al disfrute del alumnado, instando a partir sin una referencia clara sobre el tema; la ausencia de un instrumento de evaluación del disfrute para el alumnado de Educación Infantil; la escasa muestra y el tiempo disponible debido al contexto de realización del trabajo. Los datos recogidos en relación con la percepción del disfrute a través de los descansos activos por parte del alumnado pueden no ser concluyentes, pero permiten tener 
una primera impresión acerca de cómo ha resultado la implementación de esta metodología en un grupo de Educación Infantil, pudiendo ser tomado como base para futuros estudios.

\section{REFERENCIAS BIBLIOGRÁFICAS}

1. Ryan, R.M., y Deci, E.L. (2000). Self-determination theory and the facilitation of intrinsic motivation, social development and well-being. American Psychologist, 55(1), 68-78. Recuperado de https://selfdeterminationtheory.org/SDT/documents/2000_ RyanDeci_SDT.pdf.

2. Davison, K.K., Werder, J.L., Trost, S.G., Baker, B.L., y Birch, L.L. (2007). Why are early maturing girls less active? Links between pubertal development, psychological well-being and physical activity among girls at ages 11 and 13. Social Science \& Medicine, 64(12), 2391- 2404. Recuperado de https://www.ncbi.nlm.nih.gov/pmc/ articles/PMC2067993/pdf/nihms25394.pdf.

3. Fernández, E., Sánchez, B., y Salinero, J. J. (2008). Validación y adaptación de la escala PACES de disfrute con la practica de la actividad física para adolescentes españolas. Psicothema, 20(4), 890-895. Recuperado de http://www.psicothema.com/ pdf/3571.pdf.

4. Hashim, H., Grove, J. R. y Whipp, P. (2008). Validating the youth sport enjoyment construct in high school physical education. Research Quarterly for Exercise and Sport, 79(2), 183-194. Recuperado de https://www.researchgate.net/publication/23134637_ Validating_the_Youth_Sport_Enjoyment_Construct_in_High_School_Physical_Education/download.

5. Nahas, M.V., Goldfine, B., y Collins, M.A. (2003). Determinants of physical activity in adolescents and young adults. Physical Educator, 60(1), 42-56. Recuperado de https://www.researchgate.net/publication/235913873_Determinants_of_Physical_ Activity_in_Adolescents_and_Young_Adults_The_Basis_for_High_School_and_ College_Physical_Education_to_Promote_Active_Lifestyles.

6. Subramanian, P.R., y Silverman, S. (2000). Validation of scores from an instrument assessing student attitude toward physical education. Measurement in Physical Education \& Exercise Science, 4(1), 29-43. Recuperado de https://www.researchgate. net/publication/243666788_Validation_of_Scores_From_an_Instrument_Assessing_Student_Attitude_Toward_Physical_Education/downloād.

7. Pérez López, J. A. (1991). Teoría de la acción humana en las organizaciones. Madrid: Ediciones Rialp.

8. Reeve, J. (1994). Motivación y emoción. Madrid: Mc Graw-Hill, 2-235.

9. Navarrete, B. (2009). La motivación en el aula. Funciones del profesor para mejorar la motivación en el aprendizaje. Revista digital. Innovación y experiencias educativas, 15, 2-3. Recuperado de https://archivos.csif.es/archivos/andalucia/ensenanza/ revistas/csicsif/revista/pdf/Numero_15/BELEN_NAVARRETE_1.pdf.

10. Sánchez, M., Gutiérrez, D., Ruíz de la Hermosa, A., López, C. y Sánchez, M.A. (2017). Proyectos Escolares Saludables. Descansos activos. Guía para profesores. Recuperado de https://ruidera.uclm.es/xmlui/bitstream/handle/10578/13451/Proyectos_escolares_saludables.pdf?sequence $=1$ \&isAllowed=y. 
11. Mitchell, J. (2016, 31 de octubre). GoNoodle Is Creating A World Where Kids Use Technology To Unplug. Forbes.

12. Moreno, J. A., González-Cutre, D., Martínez Galindo, C., Alonso, N., y López, M. (2008). Propiedades psicométricas de la Physical Activity Enjoyment Scale (PACES) en el contexto español. Estudios de Psicología, 29(2), 173-180.

13. Blanco, J. (2014). Evaluación de la motivación hacia el aprendizaje en niños de 2 a 3 años. International Journal of Developmental and Educational Psychology, 6(1), 259266. Recuperado de https://www.redalyc.org/html/3498/349851790030/index.html.

14. Suárez-Manzano, S., Ruíz-Ariza, A., López-Serrano, S. y Martínez, E. (2018) Descansos activos para mejorar la atención en clase: intervenciones educativas. Profesorado, Revista de Currículum y Formación del Profesorado, 22(4), 287-304. Recuperado de http://revistaseug.ugr.es/index.php/profesorado/article/view/8417/7151.

15. Ruíz, J. R., Castro-Pinero, J., Artero, E. G., Ortega, F. B., Sjöström, M., Suni, J., y Castillo, M. J. (2009). Predictive validity of health-related fitness in youth: a systematic review. British journal of sports medicine, 43(12), 909-923. Recuperado de https:// pdfs.semanticscholar.org/3d14/b38d6fe5525f8e938750777682766201a45a.pdf.

16. Huang, T., Larsen, K. T., Ried-Larsen, M., Moller, N. C., y Andersen, L. B. (2014). The effects of physical activity and exercise on brain-derived neurotrophic factor in healthy humans: A review. Scandinavian Journal of Medicine \& Science in Sports, 24(1), 1-10. Recuperado de http://doi.org/10.1111/sms.12069.

17. Leckie, R. L., Oberlin, L. E., Voss, M. W., Prakash, R. S., Szabo-Reed, A., ChaddockHeyman, L., y Erickson, K. I. (2014). BDNF mediates improvements in executive function following a 1-year exercise intervention. Frontiers in Human Neuroscience, 8, 12. Recuperado de https://www.researchgate.net/publication/269632144_BDNF_ mediates_improvements_in_executive_function_following_a_1-year_exercise_intervention/download.

18. Martínez-López, J., De la Torre-Cruz, M. J. y Ruíz-Ariza, A. (2018). Capítulo 1: “Active-Breaks": una propuesta innovadora de descansos activos entre clases en Educación Secundaria. En P. Murillo y C. Gallego (Ed.), Innovación en la Práctica Educativa (pp. 13-19). Camas, Sevilla: Ediciones Egregius. 